\title{
Tear and aqueous humour cytokine profile in primary open-angle glaucoma
}

\author{
Barbara Burgos-Blasco, (D) Beatriz Vidal-Villegas, Federico Saenz-Frances, Laura Morales-Fernandez, \\ Lucia Perucho-Gonzalez, Julian Garcia-Feijoo and Jose M Martinez-de-la-Casa
}

Servicio de Oftalmología, Hospital Clínico San Carlos, Instituto de Investigación Sanitaria del Hospital Clínico San Carlos (IdISSC), Madrid, Spain

\begin{abstract}
.
Purpose: To evaluate the concentrations of pro-inflammatory cytokines in tear and aqueous humour of patients with primary open-angle glaucoma (POAG), relative to healthy controls.

Method: Tear and aqueous humour samples were collected from 29 healthy controls and 27 POAG patients. Twenty-seven inflammatory cytokines were analysed: interleukin (IL)-1ß, IL-1ra, IL-2, IL-4, IL-5, IL-6, IL-7, IL-8, IL9, IL-10, IL-12, IL-13, IL-15, IL-17, eotaxin, fibroblast growth factor (FGF) basic, granulocyte colony-stimulating factor, granulocyte-monocyte colonystimulating factor, interferon (IFN)- $\gamma$, interferon gamma-induced protein, monocyte chemo-attractant protein-1, macrophage inflammatory protein (MIP)-1 $\alpha$, MIP-1 $\beta$, platelet-derived growth factor, regulated on activation normal $T$ cell expressed and secreted, tumour necrosis factor (TNF)- $\alpha$ and vascular endothelial growth factor (VEGF).

Results: In tear samples of glaucoma patients, an increase in IL-4, IL-12, IL-15, FGF-basic and VEGF was observed, as well as a decrease in MIP-1a relative to the control group $(p<0.05)$. IL-5, IL-12, IL-15, IFN- $\gamma$ and MIP-1a were significantly higher in aqueous humour of glaucoma eyes $(p<0.05)$. A poor correlation between cytokine levels in tear and aqueous humour was observed. Conclusion: The different profiles of inflammatory marker expression of patients with POAG and healthy controls confirm the inflammatory activity of the pathology, indicating that some of them could be used as potential biomarkers of this disease.
\end{abstract}

Key words: cytokine - glaucoma - inflammation - primary open-angle glaucoma

Acta Ophthalmol.

(c) 2020 Acta Ophthalmologica Scandinavica Foundation. Published by John Wiley \& Sons Ltd

doi: $10.1111 /$ aos. 14374

\section{Introduction}

Glaucoma is an optic neuropathy, which results from the acquired loss of the retinal ganglion cells, and their axons, which form the optic nerve. This progressive loss results in alterations of the optic nerve head and consequent characteristic visual field losses. It is the second most common cause of by a relatively long delay in the death of retinal ganglion cells and by considerable interindividual variability. However, models of retinal ganglion cell death in POAG have revealed that inflammatory cytokines can directly link intraocular pressure (IOP) and ischaemia with the loss of these cells (Vohra et al. 2013; Weinreb et al. 2014).

Inflammation occurs in response to an ischaemic lesion. An acute and prolonged inflammatory process results in the release of pro-inflammatory mediators and the infiltration of inflammatory cells within the ischaemic tissue. In addition, the probable relationship of the blood-retinal barrier with glaucoma pathogenesis suggests that inflammatory responses may affect retinal ganglion cells by inducing proapoptotic reactions (Pantalon \& Constantinescu, 2019). This is more likely to occur in stress situations, such as an increase in IOP or a decrease in ocular perfusion due to low blood pressure. Disturbed self-regulation produces periods of relative ischaemia leading to reperfusion damage when normal flow is restored. As a result, concentrations of inflammatory molecules could increase within the affected areas (Vohra et al. 2013).

Previous studies have identified several molecular pathways involved in the pathogenesis of glaucoma, such as stress response, apoptosis, DNA repair, cell adhesion, tissue remodelling, transcription regulation, resistance to multiple drugs and energy metabolism. The role of inflammation in the pathogenesis of POAG is not well understood and requires further 
investigation, especially with regard to biomarkers. Tears represent one of the most accessible and non-invasive sources from which biomarkers could be assayed for glaucoma (Agnifili et al. 2015). The discovery of potential inflammatory biomarkers in tears, and correlation of these cytokines in the aqueous humour, could provide essential information about the pathophysiology of the disease and the effects of topical medications on inflammatory processes (Pinazo-Durán et al. 2013).

The primary objective of this study was to evaluate the concentrations of pro-inflammatory cytokines in tear and aqueous humour of patients with POAG in comparison with healthy controls. The secondary objective was to examine the degree of correlation and concordance between the values of cytokines in tears and in aqueous humour in both patients with POAG and healthy controls.

\section{Methods}

This cross-sectional study was conducted at the Hospital Clínico San Carlos in Madrid. Patients diagnosed with glaucoma and candidates for trabeculectomy or phacotrabeculectomy were recruited. Healthy patients undergoing cataract surgery were used as controls and matched for age and sex. The study was approved by the Clinical Research Ethics Committee and was conducted in accordance with the Helsinki Declaration. Written informed consent was obtained from all patients.

The inclusion criteria of the study group were over 40 years of age; patient candidate for trabeculectomy or phacotrabeculectomy; and diagnosis of chronic POAG. All POAG patients included in the present study required filtering surgery due to IOPs not controlled by antiglaucoma drugs. Therefore, patients with glaucoma were on topical antiglaucomatous eye drops when the samples were collected.

The inclusion criteria of the control group were over 40 years of age; absence of concomitant ophthalmological pathology (other than cataract); and patient candidate for cataract surgery. The control group consisted of patients with age-related cataracts who underwent routine cataract surgeries without a history of other eye diseases or IOP exceeding $21 \mathrm{mmHg}$.
The exclusion criteria were prior ocular surgery other than cataract surgery; cataract surgery or an ocular laser procedure in the six months prior to inclusion in the study; another type of glaucoma other than chronic POAG; concomitant ophthalmological pathology; and concomitant topical treatment other than antiglaucomatous eye drops.

On the day of surgery, the tear sample $(3-5 \mu 1)$ was collected by means of a sterile $10-\mu$ capillary tube that was placed in the inferior conjunctival fornix without provoking reflex stimulation. The aqueous humour samples $(40-50 \mu 1)$ were collected through a 30 $\mathrm{G}$ anterior chamber cannula and a 1-ml syringe under aseptic measures as the first step of the surgery, without introducing any substance previously in the anterior chamber and without contacting the iris or the limbal blood vessels.

\section{Cytokine determination}

Cytokine concentrations in the tear and aqueous humour samples were determined by the Bio-Plex Pro Human Cytokine 27-Plex Immunoassay kit (Bio-Rad Laboratories, Hercules, CA, USA). This technique is performed on a platform composed of fluorescent magnetic surfaces that allows the simultaneous detection of up to 96 samples. With this technology, 27 pro-inflammatory cytokines were quantified in a single analysis: interleukin (IL)-1 $\beta$, IL-1 ra, IL2, IL-4, IL-5, IL-6, IL-7, IL-8, IL9, IL-10, IL-12, IL-13, IL-15, IL-17, eotaxin, basic fibroblast growth factor (FGF-b), granulocyte colony-stimulating factor (G-CSF), granulocyte-macrophage colony-stimulating factor (GM-CSF), interferon gamma (IFN- $\gamma$ ), interferon gamma-induced protein (IP-10), monocytic chemotactic protein (MCP-1), macrophage inflammatory protein (MIP)-1 $\alpha$, MIP-1 $\beta$, platelet-derived growth factor (PDGF), regulated on activation, normal $\mathrm{T}$ cell expressed and secreted (RANTES), tumour necrosis factor (TNF)- $\alpha$ and vascular endothelial growth factor (VEGF). $50 \mu \mathrm{l}$ of each sample was dispensed into the microwells of each platform and read simultaneously with a Luminex MAGPIX reader (Luminex Corporation, Austin, TX, USA). Cytokine concentrations were determined by interpolating the measured fluorescence intensities emitted from each sample against their standard curves. Bio-Plex Manager ${ }^{\mathrm{TM}}$ software (Bio-Rad Laboratories, Hercules, CA, USA) was used to calculate the concentrations of the 27 cytokines.

\section{Statistics}

The data were processed and analysed statistically using IBM SPSS Statistics for Mac, version 22.0 (IBM Corp., Armonk, NY, USA). For categorical variables, frequency distributions were calculated and compared using the chi-square test. The data are represented as means and standard deviation.

To analyse the differences in the concentrations of each cytokine between the different subgroups, a Mann-Whitney $U$ test was performed. The correlation between two parameters was determined by Spearman correlation. A p-value $<0.05$ was considered to be statistically significant.

\section{Results}

The overall study population included 54 participants (56 eyes), distributed in the following groups: patients diagnosed with POAG (POAG, $n=27$ ); and a control group of healthy participants (CG, $n=29$ ). In the POAG group, five patients were on prostaglandins; six on prostaglandins and beta blockers; two on prostaglandins and carbonic anhydrase inhibitors; three on beta blockers and carbonic anhydrase inhibitors; eight on prostaglandins, beta blockers and carbonic anhydrase inhibitors; one on prostaglandins, beta blockers and $\alpha 2$ adrenergic agonists; and two on prostaglandins, beta blockers, carbonic anhydrase inhibitors and $\alpha 2$-adrenergic agonists.

In the tear samples, a statistically significant increase in IL-4, IL-12, IL15, FGF-b and VEGF was observed, as well as a significant decrease in MIP$1 \alpha$, in comparison with the control group (Table 1). In aqueous humour, a statistically significant increase of the following cytokines was observed in glaucoma patients compared to the control group: IL-5, IL-12, IL-15, IFN- $\gamma$ and MIP-1 $\alpha$ (Table 2).

The correlation of tear cytokine and aqueous humour levels in patients with POAG and the control group is shown in Tables 3 and 4.

Most of the cytokines found statistically significant in tear and aqueous humour in patients with POAG were 
Table 1. Cytokine profile in tear samples. Data are expressed as mean $(\mathrm{pg} / \mathrm{mL}) \pm$ standard deviation (SD).

\begin{tabular}{|c|c|c|c|}
\hline Tear cytokines & $\mathrm{POAG}($ mean $\pm \mathrm{SD})$ & $\mathrm{CG}($ mean $\pm \mathrm{SD})$ & $\mathrm{p}$ \\
\hline IL-1b & $5.34 \pm 1.83$ & $4.17 \pm 0.47$ & 0.480 \\
\hline IL-1RA & $28115.80 \pm 8149.84$ & $10617.76 \pm 3991.02$ & 0.393 \\
\hline IL-2 & $18.69 \pm 2.76$ & $13.02 \pm 3.03$ & 0.409 \\
\hline IL-4 & $3.77 \pm 0.41$ & $1.10 \pm 0.50$ & $0.029 *$ \\
\hline IL-5 & $41.34 \pm 5.97$ & $33.43 \pm 6.30$ & 0.421 \\
\hline IL-6 & $33.07 \pm 10.26$ & $8.77 \pm 1.01$ & 0.277 \\
\hline IL-7 & $36.20 \pm 8.62$ & $42.05 \pm 7.34$ & 0.378 \\
\hline IL-8 & $642.01 \pm 411.71$ & $369.18 \pm 111.34$ & 0.310 \\
\hline IL-9 & $6.59 \pm 2.13$ & $3.02 \pm 0.00$ & 0.207 \\
\hline IL-10 & $10.71 \pm 3.16$ & $14.26 \pm 3.14$ & 0.293 \\
\hline IL-12 & $38.83 \pm 4.14$ & $24.54 \pm 4.35$ & $0.038^{*}$ \\
\hline IL-13 & $4.35 \pm 1.07$ & $6.47 \pm 1.18$ & 0.162 \\
\hline IL-15 & $1069.27 \pm 91.44$ & $414.95 \pm 144.43$ & $0.001 * *$ \\
\hline IL-17 & $32.71 \pm 5.84$ & $26.78 \pm 5.13$ & 0.201 \\
\hline Eotaxin 1 & $12.29 \pm 1.78$ & $16.10 \pm 4.54$ & 0.549 \\
\hline FGF-b & $232.05 \pm 31.91$ & $125.28 \pm 35.22$ & $0.008 * *$ \\
\hline G-CSF & $371.32 \pm 194.52$ & $272.92 \pm 80.47$ & 0.472 \\
\hline GM-CSF & $8.24 \pm 4.39$ & $4.44 \pm 0.84$ & 0.521 \\
\hline IFN-Y & $66.21 \pm 20.45$ & $73.21 \pm 13.95$ & 0.156 \\
\hline IP-10 & $47563.12 \pm 9134.23$ & $36197.72 \pm 8161.13$ & 0.370 \\
\hline MCP-1 & $268.36 \pm 36.38$ & $207.33 \pm 41.21$ & 0.152 \\
\hline MIP-1 $1 \alpha$ & $6.87 \pm 5.89$ & $8.62 \pm 1.22$ & $0.008 * *$ \\
\hline PDGF & $112.83 \pm 22.77$ & $72.38 \pm 18.48$ & 0.189 \\
\hline MIP-1 $\beta$ & $32.30 \pm 15.94$ & $18.78 \pm 2.36$ & 0.167 \\
\hline RANTES & $58.27 \pm 12.89$ & $69.02 \pm 9.41$ & 0.327 \\
\hline TNF- $\alpha$ & $42.72 \pm 7.10$ & $28.59 \pm 5.48$ & 0.094 \\
\hline VEGF & $3082.25 \pm 543.33$ & $1327.28 \pm 475.60$ & $0.006^{* *}$ \\
\hline
\end{tabular}

$\mathrm{CG}=$ control group, $\mathrm{POAG}=$ primary open-angle glaucoma. Statistically significant differences:

$*(\mathrm{p}<0.05) ; * *(\mathrm{p}<0.01)$.

Table 2. Cytokine profile in aqueous humour. Data are expressed as mean $(\mathrm{pg} / \mathrm{mL}) \pm$ standard deviation (SD).

\begin{tabular}{lccl}
\hline Aqueous humour cytokines & POAG $($ mean $\pm \mathrm{SD})$ & $\mathrm{CG}(\mathrm{mean} \pm \mathrm{SD})$ & $\mathrm{p}$ \\
\hline IL-1b & $1.44 \pm 0.29$ & $1.78 \pm 0.39$ & 0.756 \\
IL-1RA & $16054.81 \pm 7905.07$ & $1869.69 \pm 547.49$ & 0.199 \\
IL-2 & $3.52 \pm 1.07$ & $1.92 \pm 0.83$ & 0.093 \\
IL-4 & $0.79 \pm 0.21$ & $0.39 \pm 0.08$ & 0.192 \\
IL-5 & $36.03 \pm 8.05$ & $16.21 \pm 4.26$ & $0.043^{*}$ \\
IL-6 & $6.41 \pm 1.09$ & $4.93 \pm 1.07$ & 0.194 \\
IL-7 & $13.61 \pm 5.44$ & $9.10 \pm 1.97$ & 0.767 \\
IL-8 & $227.16 \pm 104.63$ & $68.84 \pm 24.89$ & 0.323 \\
IL-9 & $208.96 \pm 53.08$ & $33.23 \pm 11.16$ & 0.156 \\
IL-10 & $19.54 \pm 4.38$ & $11.85 \pm 3.69$ & 0.096 \\
IL-12 & $27.56 \pm 4.84$ & $13.39 \pm 3.92$ & $0.018^{*}$ \\
IL-13 & $1.02 \pm 0.29$ & $1.86 \pm 0.73$ & 0.735 \\
IL-15 & $839.02 \pm 123.09$ & $601.91 \pm 145.38$ & $0.017^{*}$ \\
IL-17 & $18.86 \pm 3.49$ & $15.01 \pm 5.29$ & 0.138 \\
Eotaxin & $10.41 \pm 1.01$ & $8.08 \pm 0.96$ & 0.066 \\
FGF-b & $28.18 \pm 4.91$ & $21.62 \pm 3.38$ & 0.192 \\
G-CSF & $276.19 \pm 59.85$ & $142.51 \pm 55.50$ & 0.28 \\
GM-CSF & $5.81 \pm 2.84$ & $9.14 \pm 3.05$ & 0.274 \\
IFN-Y & $51.27 \pm 12.87$ & $29.46 \pm 8.97$ & $0.026^{*}$ \\
IP-10 & $25466.90 \pm 13841.91$ & $12539.34 \pm 4910.37$ & 0.156 \\
MCP-1 & $203.49 \pm 24.31$ & $215.55 \pm 23.35$ & 0.642 \\
MIP-1 $\alpha$ & $4.99 \pm 2.86$ & $1.29 \pm 0.35$ & $0.016^{*}$ \\
PDGF & $87.40 \pm 20.23$ & $63.48 \pm 26.16$ & 0.119 \\
MIP-1 $\beta$ & $12.55 \pm 2.78$ & $10.12 \pm 1.66$ & 0.463 \\
RANTES & $70.07 \pm 12.92$ & $51.46 \pm 37.30$ & 0.057 \\
TNF- $\alpha$ & $24.77 \pm 8.17$ & $17.33 \pm 4.66$ & 0.904 \\
VEGF & $1544.54 \pm 345.34$ & $1417.63 \pm 4.66$ & 0.73 \\
\hline
\end{tabular}

$\mathrm{CG}=$ control group, POAG= primary open-angle glaucoma. Statistically significant differences: $*(\mathrm{p}<0.05)$.
Table 3. Correlation of cytokine concentration in aqueous humour with tear concentrations in $\mathrm{CG}$.

\begin{tabular}{|c|c|c|}
\hline Cytokines & Correlation & $\mathrm{p}$ \\
\hline IL-1b & 0.102 & 0.571 \\
\hline IL-1RA & 0.031 & 0.864 \\
\hline IL-2 & -0.149 & 0.406 \\
\hline IL-4 & -0.562 & $0.001 * *$ \\
\hline IL-5 & -0.368 & $0.035^{*}$ \\
\hline IL-6 & -0.391 & $0.025^{*}$ \\
\hline IL-7 & -0.28 & 0.114 \\
\hline IL-8 & 0.339 & 0.053 \\
\hline IL-9 & - & - \\
\hline IL-10 & -0.208 & 0.246 \\
\hline IL-12 & -0.266 & 0.135 \\
\hline IL-13 & -0.189 & 0.293 \\
\hline IL-15 & -0.664 & $0.001 * *$ \\
\hline IL-17 & -0.333 & 0.058 \\
\hline Eotaxin 1 & -0.068 & 0.709 \\
\hline FGF-b & -0.514 & $0.002 * *$ \\
\hline G-CSF & -0.344 & $0.050 *$ \\
\hline GM-CSF & -0.405 & $0.019^{*}$ \\
\hline IFN-Y & -0.331 & 0.06 \\
\hline IP-10 & -0.342 & 0.052 \\
\hline MCP-1 & -0.16 & 0.375 \\
\hline MIP-1 $1 \alpha$ & -0.173 & 0.336 \\
\hline PDGF & -0.398 & $0.022^{*}$ \\
\hline MIP-1 $\beta$ & -0.21 & 0.241 \\
\hline RANTES & 0.162 & 0.369 \\
\hline TNF- $\alpha$ & -0.337 & 0.055 \\
\hline VEGF & -0.502 & $0.003^{* *}$ \\
\hline
\end{tabular}

Statistically significant differences: $*(p<0.05)$; $* *(\mathrm{p}<0.01)$.

not correlated with the number of topical medications (Table 5).

\section{Discussion}

This study's main objective was to profile mediators of inflammation in the tears and aqueous humour of patients diagnosed with POAG in comparison with non-POAG cataract patients (control group). To this end, sensitive immunoassays were performed on a panel of 27 cytokines. Our results demonstrate a differential expression of the main inflammation markers in patients with POAG compared to control subjects. IL-4, IL-12, IL-15, FGF-b and VEGF were increased in the tears of patients with POAG and decreased abundance of MIP- $1 \alpha$ was observed. In aqueous humour, a relative increase in IL-5, IL-12, IL-15, IFN- $\gamma$ and MIP- $1 \alpha$ was found in POAG patients.

Since the involvement of inflammation in POAG was first reported, several research groups have analysed inflammatory cytokines in tear and aqueous humour samples. For 
Table 4. Correlation of cytokine concentration in aqueous humour with tear concentrations in patients with primary open-angle glaucoma.

\begin{tabular}{|c|c|c|}
\hline Cytokines & Correlation & $\mathrm{p}$ \\
\hline IL-1b & -0.223 & 0.245 \\
\hline IL-1RA & -0.009 & 0.961 \\
\hline IL-2 & 0.193 & 0.315 \\
\hline IL-4 & -0.191 & 0.32 \\
\hline IL-5 & -0.257 & 0.178 \\
\hline IL-6 & 0.276 & 0.148 \\
\hline IL-7 & 0.123 & 0.524 \\
\hline IL-8 & 0.029 & 0.882 \\
\hline IL-9 & -0.33 & 0.08 \\
\hline IL-10 & -0.154 & 0.426 \\
\hline IL-12 & -0.205 & 0.285 \\
\hline IL-13 & 0.14 & 0.469 \\
\hline IL-15 & -0.327 & 0.084 \\
\hline IL-17 & 0.183 & 0.343 \\
\hline Eotaxin 1 & -0.034 & 0.861 \\
\hline FGF-b & -0.469 & $0.010^{*}$ \\
\hline G-CSF & 0.156 & 0.418 \\
\hline GM-CSF & -0.094 & 0.629 \\
\hline IFN-Y & -0.064 & 0.74 \\
\hline IP-10 & 0.482 & $0.008 * *$ \\
\hline MCP-1 & -0.364 & 0.052 \\
\hline MIP-1 $\alpha$ & 0.203 & 0.29 \\
\hline PDGF & -0.481 & $0.008 * *$ \\
\hline MIP-1 $\beta$ & 0.169 & 0.381 \\
\hline RANTES & -0.327 & 0.083 \\
\hline TNF- $\alpha$ & -0.065 & 0.736 \\
\hline VEGF & -0.452 & $0.014^{*}$ \\
\hline
\end{tabular}

Statistically significant differences: $*(p<0.05)$; $* *(\mathrm{p}<0.01)$.

Table 5. Spearman correlation of cytokine concentration in tear and aqueous humour with number of topical hypotensors in patients with primary open-angle glaucoma.

\begin{tabular}{lcl}
\hline Cytokines & Correlation & $\mathrm{p}$ \\
\hline In tear & & \\
IL-4 & 0.197 & 0.31 \\
IL-12 & -0.297 & 0.12 \\
IL-15 & -0.075 & 0.7 \\
FGF-b & 0.001 & 0.99 \\
MIP-1 $\alpha$ & -0.283 & 0.14 \\
VEGF & -0.087 & 0.65 \\
In aqueous humour & \\
IL-5 & 0.252 & 0.19 \\
IL-12 & 0.379 & $0.04 *$ \\
IL-15 & 0.14 & 0.47 \\
IFN- $\Upsilon$ & 0.315 & 0.10 \\
MIP-1 $\alpha$ & 0.148 & 0.44 \\
\hline
\end{tabular}

Statistically significant differences: $*(\mathrm{p}<0.05)$.

example, differences in IL-6 concentrations in aqueous humour has been described in patients with POAG compared to controls. Given that IL-6 is a crucial mediator of endothelial dysfunction and enhances vascular permeability, increased IL-6 could contribute to the dysfunction of the blood- aqueous barrier in patients with $\mathrm{POAG}$ (Khalef et al. 2017; Benitez-del-Castillo et al. 2019). However, our results do not show statistically significant differences in IL-6 abundance.

Multiple studies describe a relative increase in IL-8 in patients with POAG. IL- 8 is a major chemotactic cytokine for neutrophils that has been found to accumulate in the trabecular meshwork in POAG. Our data showed an increased abundance of IL- 8 in both tear and aqueous humour, but these results did not achieve the level of statistical significance. Ten Berge et al. found that the highest levels of IL- 8 in glaucoma patients were in those that had undergone prior surgical treatment (ten Berge et al. 2019). This suggests that higher levels of IL-8 may result from immune activation in response to tissue damage caused by previous surgery. Given that increased IL-8 has been consistently described in different types of glaucoma with and without inflammation, it has been postulated that IL-8 could be involved in the pathogenesis of glaucoma or greater abundance could be a consequence of higher IOP. However, it has not been possible to demonstrate causality and better understanding of how this cytokine is involved in glaucoma is needed (Khalef et al. 2017; Benitez-del-Castillo et al. 2019):; Kokubun et al. 2018).

The relative abundance of TNF- $\alpha$ has also been reported to be increased in glaucoma patients (Kondkar et al. 2018). In this study, patients with POAG have relatively higher concentrations of this cytokine in both tear and aqueous humour, but this result was below statistical significance $(\mathrm{p}=0.09)$. Higher abundance of TNF- $\alpha$ in the retina and optic nerve of glaucoma patients, as well as in plasma, has been described. Based on these findings, it was postulated that TNF- $\alpha$ is involved in the apoptosis of the retinal ganglion cells and not in the increase of the outflow resistance (Khalef et al. 2017; ten Berge et al. 2019; Tong et al. 2017).

IFN- $\gamma$ is secreted by helper $\mathrm{T}$ cells, cytotoxic $\mathrm{T}$ cells and natural killer cells and has antiviral, immunoregulatory and anti-tumour properties. Csösz hypothesized that increased IFN- $\gamma$ may inhibit collagen synthesis and lead to delayed epidermal wound healing (Csősz et al. 2019).

VEGF is a cytokine associated with neovascularization, inflammation and remodelling of the drainage system. It has been postulated that VEGF expression correlates with the aggressiveness of glaucoma and its refractoriness to treatment. This cytokine has been correlated with the levels of multiple cytokines in neovascular glaucoma, suggesting that this pro-inflammatory cytokine may have a determining role in the promotion of this disease (Kokubun et al. 2018).

Other cytokines, such as IL-2, IL-4, IL-5, IL-12 and IL-17, have also been found to be increased in patients with POAG but are less studied (Tong et al., 2017; Chono et al., 2018; Kokubun et al., 2018).

The levels of IL-1 $\beta$, IL-1ra, IL-4, IL-7, MCP-1 and TNF- $\alpha$ in aqueous humour were in the same range as previous research which utilized similar methods in POAG patients, whereas the levels of IL-2, IL-6, FGF-b, GM-CSF, IL-13, IL17 and MIP-1 $\alpha$ were lower in our study. At the same time, the levels of IL-5, eotaxin, G-CSF, IFN- $\gamma$, IL-8, IL-9, IL10, IL-12, IL-15, IP10, MIP-1 $\beta$, PDGF, RANTES and VEGF were higher in our study compared to the studies reported by Csősz et al. (2019). However, cytokine levels may be difficult to compare among different research groups due to, for example, variability in stages of the disease of the enrolled patients, differences in age, alternative profiles of pharmacological treatment and different analytical assays for cytokines (Duvesh et al. 2017; Garweg et al. 2017; Martinezde-la-Casa et al. 2017). Nonetheless, all studies, including the present one, have showed a unique inflammatory response profile in the aqueous humour of patients with POAG compared to control subjects (Martinez-de-la-Casa et al. 2017; Csősz et al. 2019).

To the best of our knowledge, only Csosz's study group has analysed the correlation between cytokines in tear and aqueous humour in POAG patients, and our study was the first one to compare these results with healthy patients. Csosz et al. described significantly higher levels of all studied cytokine and chemokine molecules, except IL-2, in tears compared to aqueous humour. Our results showed a poor correlation in the levels of cytokines between both ocular fluids in the majority of cases. In those instances where a statistically significant correlation was detected, cytokine levels in tears were higher than in aqueous humour, in agreement with 
Csosz et al. (Csősz et al. 2019). Topical treatment and other external agents may affect the expression of inflammatory cytokines (Martinez-de-la-Casa et al. 2017; Reddy et al. 2018) and mask the true inflammatory nature of POAG. To account for this, it would be necessary to compare the concentrations of cytokines in aqueous humour and tears in a larger POAG population which is grouped according to pharmacological treatment and severity of the disease.

An important limitation of cytokine tear concentrations in this study was the patients' topical hypotensive treatment. It was not possible to perform a previous washout of the treatment due to ethical reasons, since patients were diagnosed with advanced glaucoma and required surgery to prevent further disease progression. In addition, a 1month washout period, for example, would not guarantee the absence of an artefact in cytokine concentrations because of the drops. Furthermore, in accordance with our study, other research groups have not performed a topical treatment washout.

To investigate the possible effect of topical treatment on cytokine concentrations, the correlation between cytokine abundance in tear and aqueous humour of glaucoma patients with the number of hypotensive drops was analysed. Given the limited number of patients, an analysis could not be performed based on the type of treatment. When comparing the number of eye drops with cytokine levels, no large differences in the inflammatory profile were found, with the exception that only IL-12 in aqueous humour was statistically significant (correlation $0.379, \mathrm{p}=0.042$ ). Engel et al. (2014) found a correlation in cytokine abundance of patients with POAG with and without previous surgery. However, when comparing patients with and without topical treatment, they did not observe significant differences between the cytokine profiles. These data support our hypothesis that cytokine concentrations are poorly affected by topical treatment and that glaucoma truly is an inflammatory pathology.

In conclusion, non-invasive and invasive methods were used to obtain tear and aqueous humour samples, respectively, from POAG and control patients. The expression of inflammatory cytokines was quantified using an immunoassay system based on flow cytometry. Our results show differences in the profiles of inflammatory proteins which suggests that some of them could be used as biomarkers of this disease. However, no correlation or concordance between the values of tear cytokines and aqueous humour in patients with POAG was found in the current study, which suggests that the levels of tear cytokines can be altered by multiple causes.

\section{References}

Agnifili L, Pieragostino D, Mastropasqua A, Fasanella V, Brescia L, Tosi GM \& Mastropasqua L. (2015): Molecular biomarkers in primary open-angle glaucoma: From noninvasive to invasive. Prog Brain Res 221: 1-32.

Benitez-del-Castillo J, Cantu-Dibildox J, SanzGonzález SM, Zanón-Moreno V \& PinazoDuran MD (2019): Cytokine expression in tears of patients with glaucoma or dry eye disease: A prospective, observational cohort study. Eur J Ophthalmol 29: 437-443.

ten Berge JC, Fazil Z, van den Born I, Wolfs RCW, Schreurs MWJ, Dik WA \& Rothova A (2019): Intraocular cytokine profile and autoimmune reactions in retinitis pigmentosa, age-related macular degeneration, glaucoma and cataract. Acta Ophthalmol 97: 185-192.

Chono I, Miyazaki D, Miyake H, Komatsu N, Ehara F, Nagase D \& Inoue Y (2018): High interleukin-8 level in aqueous humor is associated with poor prognosis in eyes with open angle glaucoma and neovascular glaucoma. Sci Rep 8: 1-11.

Csősz É, Deák E, Tóth N, Traverso CE, Csutak A \& Tőzsér J (2019): Comparative analysis of cytokine profiles of glaucomatous tears and aqueous humour reveals potential biomarkers for trabeculectomy complications. FEBS Open Bio 9: 1020-1028.

Duvesh R, Puthuran G, Srinivasan K, Rengaraj V, Krishnadas SR, Rajendrababu S \& Sundaresan P (2017): Multiplex cytokine analysis of aqueous humor from the patients with chronic primary angle closure glaucoma. Curr Eye Res 42: 1608-1613.

Engel LA, Muether PS, Fauser S \& Hueber A (2014): The effect of previous surgery and topical eye drops for primary open-angle glaucoma on cytokine expression in aqueous humor. Graefe's Arch Clin Exp Ophthalmol 252: 791-799.

Foster PJ (2002): The definition and classification of glaucoma in prevalence surveys. $\mathrm{Br}$ J Ophthalmol 86: 238-242.

Garweg JG, Zandi S, Pfister IB, Skowronska M \& Gerhardt C (2017): Comparison of cytokine profiles in the aqueous humor of eyes with pseudoexfoliation syndrome and glaucoma. PLoS ONE 12: 1-13.

Khalef N, Labib H, Helmy H, El Hamid MA, Moemen L \& Fahmy I (2017): Levels of cytokines in the aqueous humor of eyes with primary open angle glaucoma, pseudoexfoliation glaucoma and cataract. Elect Phys 9: 3833-3837.

Kokubun T, Tsuda S, Kunikata H, Yasuda M, Himori N, Kunimatsu-Sanuki S \& Nakazawa T (2018): Characteristic profiles of inflammatory cytokines in the aqueous humor of glaucomatous eyes. Ocul Immunol Inflammat 26: 1177-1188.

Kondkar A, Sultan T, Almobarak F, Kalantan H, Al-Obeidan S \& Abu-Amero K (2018): Association of increased levels of plasma tumor necrosis factor alpha with primary open-angle glaucoma. Clin Ophthalmol 12: 701-706.

Martinez-de-la-Casa JM, Perez-Bartolome F, Urcelay E, Santiago JL, Moreno-Montañes $\mathrm{J}$, Arriola-Villalobos P \& Garcia-Feijoo J (2017): Tear cytokine profile of glaucoma patients treated with preservative-free or preserved latanoprost. Ocular Surf 15: 723-729.

Pantalon A \& Constantinescu D (2019): Inflammatory model in patients with primary open angle glaucoma and diabetes. Int J Ophthalmol 12: 795-801.

Pinazo-Durán MD, Zanón-Moreno V, García-Medina JJ \& Gallego-Pinazo R (2013): Evaluation of presumptive biomarkers of oxidative stress, immune response and apoptosis in primary open-angle glaucoma. Curr Opin Pharmacol 13: 98-107.

Quigley HA (2011): Glaucoma. Lancet 377: 1367-1377.

Quigley HA \& Broman AT (2006): The number of people with glaucoma worldwide in 2010 and 2020. Brit J Ophthalmol 90: 262267.

Reddy S, Sahay P, Padhy D, Sarangi S, Suar M, Modak R \& Rao A (2018): Tear biomarkers in latanoprost and bimatoprost treated eyes. PLoS ONE 13: e0201740.

Tong Y, Zhou Y, Zheng Y, Biswal M, Zhao P \& Wang $Z$ (2017): Analyzing cytokines as biomarkers to evaluate severity of glaucoma. Int J Ophthalmol 10: 925-930.

Vohra R, Tsai JC \& Kolko M (2013): The Role of Inflammation in the Pathogenesis of Glaucoma. Surv Ophthalmol 58: 311-320.

Weinreb RN, Aung T \& Medeiros FA (2014): The pathophysiology and treatment of glaucoma: a review. JAMA 311: 1901-1911.

Received on September 10th, 2019.

Accepted on January 20th, 2020.

Correspondence:

Barbara Burgos-Blasco

C/ Prof Martin Lagos s/n

28040. Madrid

Spain

Tel: + 34913303132

Email: bburgos171@hotmail.com 